\title{
Intravenous injection of methylprednisolone reduces the incidence of postextubation stridor in intensive care unit patients*
}

\author{
Kuo-Chen Cheng, MD; Ching-Cheng Hou, MD; Heng-Ching Huang, MD; Shu-Chih Lin, RRT; \\ Haibo Zhang, MD, PhD
}

Objective: To determine whether treatment with corticosteroids decreases the incidence of postextubation airway obstruction in an adult intensive care unit.

Design: Clinical experiment.

Setting: Adult medical and surgical intensive care unit of a teaching hospital.

Patients: One hundred twenty-eight patients who were intubated for $>24$ hrs with a cuff leak volume $<24 \%$ of tidal volume and met weaning criteria.

Interventions: Patients were randomized into a placebo group (control, $n=43$ ) receiving four injections of normal saline every $6 \mathrm{hrs}$, a 4INJ group $(n=42)$ receiving four injections of methylprednisolone sodium succinate, or a $1 \mathrm{INJ}$ group $(n=42)$ receiving one injection of the corticosteroid followed by three injections of normal saline. Cuff volume was assessed $1 \mathrm{hr}$ after each injection, and extubation was performed $1 \mathrm{hr}$ after the last injec- tion. Postextubation stridor was confirmed by examination using bronchoscopy or laryngoscopy.

Measurements and Main Results: The incidences of postextubation stridor were lower both in the 1INJ and the 4INJ groups than in the control group (11.6\% and $7.1 \%$ vs. $30.2 \%$, both $p<$ $.05)$, whereas there was no difference between the two treated groups $(p=.46)$. The cuff leak volume increased after the second and fourth injection in the 4INJ group and after a second injection in the 1INJ group compared with the control group (both $p<.05$ ).

Conclusions: A reduced cuff leak volume is a reliable indicator to identify patients at high risk to develop stridor. Treatment with a single or multiple injections of methylprednisolone can effectively reduce the occurrence of postextubation stridor. (Crit Care Med 2006; 34:1345-1350)

KEY WORDS: endotracheal intubation; cuff-leak test; laryngeal edema; corticosteroids

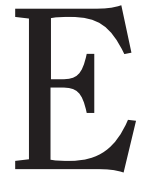

ndotracheal intubation with or without mechanical ventilation is commonly used for life support in the intensive care unit (ICU). Patients can tolerate this procedure for up to several weeks (1). However, complications resulting from intubation/ extubation such as airway mucosal ulceration, inflammation, and edema may lead to the development of postextubation stridor $(2-5)$. The incidence of postextubation stridor ranges between $2 \%$ and $16 \%$ in pa-

*See also p. 1547.

From the Department of Intensive Care Medicine, Chi Mei Medical Center, Tainan, Taiwan (K-CC, H-CH); Department of Medicine, National Defense Medical Center, Taipei, Taiwan (K-CC); Department of Intensive Care Medicine, Chi Mei Hospital, Liouying, Taiwan (C-CH); and Department of Anaesthesia, Interdepartmental Division of Critical Care Medicine, Department of Physiology, University of Toronto, St. Michael's Hospital, Toronto, Ontario, Canada (HZ).

Supported, in part, by grant CMMCR-9231 from Chi Mei Medical Center and CIHR grant MOP-69042 from the Canadian Institute of Health Research.

The authors have no financial interest to disclose in relation to this article.

Copyright (c) 2006 by the Society of Critical Care Medicine and Lippincott Williams \& Wilkins

DOI: 10.1097/01.CCM.0000214678.92134.BD tients who are endotracheally intubated for $>24$ hrs $(4,5)$. Postextubation stridor characterized by laryngeal edema can prolong ICU stay and even increase mortality rate (6-8). Thus, a reliable method to predict the occurrence of postextubation stridor and a search for effective therapies are necessary to improve quality of life and reduce ICU mortality.

The endotracheal tube cuff-leak test as an indicator of endotracheal extubation is not conclusive $(9-11)$. We speculated that repeated dynamic cuff leak tests over a period of time, however, might provide a reliable technique to predict and help prevent the occurrence of postextubation stridor.

A single-dose injection of corticosteroids $1 \mathrm{hr}$ before extubation was not effective in treating postextubation stridor (4, 12). Interestingly, treatment with multiple doses of corticosteroids, which has not been tested in adult patients, reduces the incidence of postextubation airway obstruction in pediatric patients (13). In these previous studies there was no standardized protocol with respect to the administration of corticosteroids. For example, the doses used and timing for administration were very different: a dose of $100 \mathrm{mg}$ of hydro- cortisone sodium succinate was infused intravenously $1 \mathrm{hr}$ before extubation (4); an intravenous bolus of $8 \mathrm{mg}$ of dexamethasone was given $1 \mathrm{hr}$ before extubation (12); and dexamethasone was given intravenously $(0.5 \mathrm{mg} / \mathrm{kg}$, maximal dose $10 \mathrm{mg})$ every 6 hrs for six doses, beginning 6-12 hrs before extubation (13). Also, the patient selection varied: Patients of $>15$ yrs with planned elective extubation were included (4); patients of $>15$ yrs were divided into a short period $(<36$ hrs $)$ of intubation vs. a long period ( $>36 \mathrm{hrs}$ ) (12); or pediatric patients ( $<5$ yrs) intubated for $48 \mathrm{hrs}$ were studied.

An objective of the present study was to test whether treatment with a single or multiple injections of corticosteroids for a given time frame would have any effect in reducing the incidence of postextubation stridor using the same inclusion criteria in high-risk adult ICU patients.

\section{MATERIALS AND METHODS}

Patients. Patients were recruited from an adult medical and surgical ICU at the Chi Mei Medical Center, a teaching hospital, in Taiwan between February 2002 and July 2004. The study protocol was approved by the institu- 
tional Research Ethic Board. Informed consent was obtained from the patients or their relatives before entering the study.

All patients were $>18$ yrs of age, had been intubated $>24 \mathrm{hrs}$, and met the weaning criteria defined as respiratory rate $<30$ breaths/ min, negative inspiratory force $>25 \mathrm{~cm} \mathrm{H}_{2} \mathrm{O}$, tidal volume $>5 \mathrm{~mL} / \mathrm{kg}$ of ideal body weight, and rapid shallow index $<105$ breaths/min/L.

Exclusion criteria were any treatment with corticosteroids 1 wk before extubation $(\mathrm{n}=$ $164)$, nasal or throat disease/surgery $(\mathrm{n}=9)$, gastrointestinal bleeding $(n=33)$, hyperglycemia (blood sugar $>250 \mathrm{mg} \%, \mathrm{n}=19$ ), acute cardiac attack and cardiac surgery $(n=36)$, or history of extubation during the same hospitalization course $(n=78)$. The reason to exclude patients with gastrointestinal bleeding or hyperglycemia was to better distinguish between underlying diseases and steroidsinduced complications if any.

Auscultation Cuff-Leak Test. Auscultation cuff-leak and cuff-leak volume were examined and classified into three categories: a) no leak, where no sound of leak was heard by using stethoscope detection; b) mild leak, where a leak was heard using a stethoscope; and c) significant leak, where the sound of a leak was heard without using a stethoscope.

Cuff Leak Volume. The actual tidal volume at expiration was measured before and after deflation of the endotracheal tube cuff as previously described (9). The difference in the actual tidal volume before and after cuff deflation was defined as the cuff leak volume (CLV).

Mechanical Ventilation. The patients were mechanically ventilated in a volume assistcontrol mode (Puritan-Bennett 7200 AE, Carlsbad, CA, or Bird 8400, Palm Springs, CA). The tidal volume was set at $8 \mathrm{~mL} / \mathrm{kg}$ ideal body weight with respiratory rate 20 breaths/min and zero positive end-expiratory pressure during CLV measurement.

Protocol. Patients with a CLV $\geq 24 \%$ of tidal volume during inflation were the time matching control arm for 48 hrs after extubation (nonintervention arm). This cutoff point was set based on a previous report where 13 of 110 patients who had CLVs ranging from $0 \%$ to $24 \%$ developed postextubation stridor (9).

Patients who had a CLV $<24 \%$ of tidal volume during inflation were considered at high risk to develop postextubation stridor (9, 10) and were included in the randomized, double-blinded, and placebo-controlled clinical trial (intervention arm). The randomization procedure was carried out with random numbers. The placebo and methylprednisolone had an identical volume and appearance and were packaged in an identical manner by a respiratory therapist who was not involved in the trial. Neither the physician who prescribed the drug nor the staff who administered the infusion were aware of the intervention treatment of infusion fluid.

These patients were divided into three groups: The 4INJ group received intravenous infusion of methylprednisolone sodium succi- nate (Pharmacia Upjohn, Kalamazoo, MI) at $40 \mathrm{mg}$ in $2 \mathrm{~mL}$ of normal saline every $6 \mathrm{hrs}$ over 24 hrs; the 1INJ group received one infusion of methylprednisolone sodium succinate followed by three injections of normal saline every 6 hrs over 24 hrs; and a placebo group received intravenous infusion of normal saline in $2 \mathrm{~mL}$ every $6 \mathrm{hrs}$ over 24 hrs before extubation.

Extubation was performed $1 \mathrm{hr}$ after the last injection of intervention fluid. Cuff leak volume was not used as a criterion for extubation but was used afterward for data analysis. Patients were monitored for $48 \mathrm{hrs}$ after extubation. The clinical stridor symptom was defined by the presence of an audible highpitched wheeze associated with respiratory distress requiring medical intervention (10, $14,15)$. Epinephrine inhalation and, in case of failure in response to three doses of epinephrine inhalation, bilevel positive airway pressure (Respironics, Murrysville, PA) were given to treat stridor, and reintubation was performed in patients who were not improved under epinephrine inhalation or/and bilevel positive airway pressure treatment. In the intervention arm, only patients with stridor underwent either bronchoscopy or laryngoscopy to further identify the occurrence of laryngeal edema by an in-charge physician during reintubation.

The patients in the nonintervention arm underwent extubation immediately after the cuff-leak test. In patients of the intervention arm, extubation was performed $1 \mathrm{hr}$ after the last injection of drug or placebo saline depending on the study protocol enrolled.

Statistics. Patient characteristics were analyzed using the unpaired Student's $t$-test for continuous variables. Fisher's exact test was used for analysis of categorical variables. To estimate the risk of developing postextubation stridor, logistic regression analysis was applied. In univariate analysis, crude odds ratio and a $95 \%$ confidence interval of odds ratio was estimated for each risk factor we studied. A forward stepwise method was then used to construct the best final model, which includes the risk factors significantly correlated to developing stridor and leaves out those do not improve the model. In multivariate analysis, an independent effect of each risk factor was estimated with adjusted odds ratio and 95\% confidence interval. To find an optimal cutoff value of CLV predictive of postextubation stridor, a receiver operating characteristic curve was used. We considered $p<.05$ to be statistically significant.

\section{RESULTS}

Nonintervention Arm. A total of 334 patients were included for the cuff-leak test; 13 patients were withdrawn due to deterioration of clinical conditions resulting in a delayed extubation $(\mathrm{n}=12)$ or selfextubation $(n=1)$. Thus, 321 patients were enrolled in the study protocol (Fig. 1). Of 321 patients, 193 patients who had a CLV $\geq 24 \%$ of tidal volume during inflation served as a nonintervention arm. The cutoff of $24 \%$ was based on the study by Sandhu and coworkers (9). The other 128 patients with a CLV $<24 \%$ served as an intervention arm and were randomized into a placebo group ( $n=43)$, a 4INJ group $(n=42)$, or a 1INJ group $(n=43)$. The incidence of postextubation stridor was $2.6 \%$ in the nonintervention arm and $30.2 \%$ in the placebo group of the intervention arm. This suggests that the cutoff point of $24 \%$ of CLV reliably predicts the development of postextubation stridor in this population of ICU patients.

To identify the critical point of CLV in predicting the development of postextubation stridor, we retrospectively prepared and analyzed a receiver operating characteristic curve and found that 18\% was the best predictor in our patient population in the present study (Fig. 2). There was an excellent agreement between the auscultation cuff-leak test and the CLV (Fig. 3).

Since the robustness of a multivariate logistic model depends on the inclusion of all relevant variables, we particularly examined if the level of training of the operator to intubate patients was a significant risk factor to cause postextubation stridor. It appeared that residents were the most frequent operators of intratracheal intubation

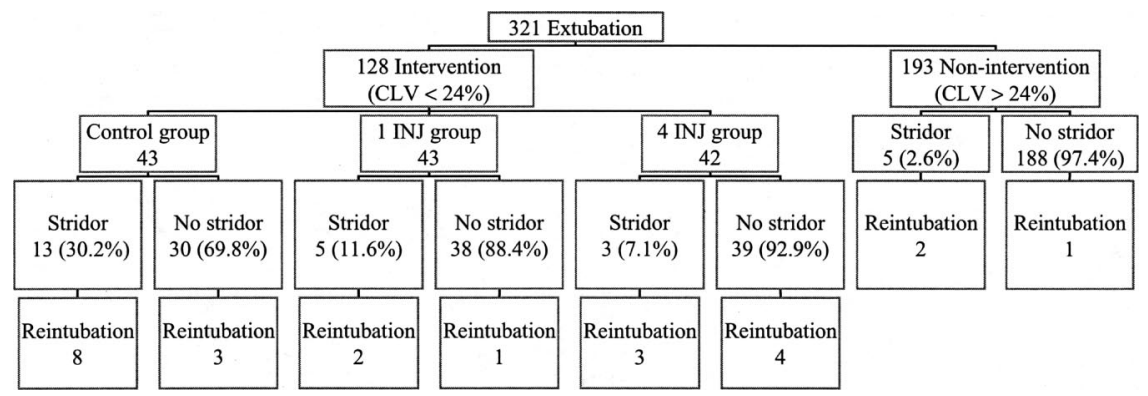

Figure 1. Distribution of groups of patients. $C L V$, cuff leak volume; IINJ, one injection of methylprednisolone; $4 I N J$, four injections. 


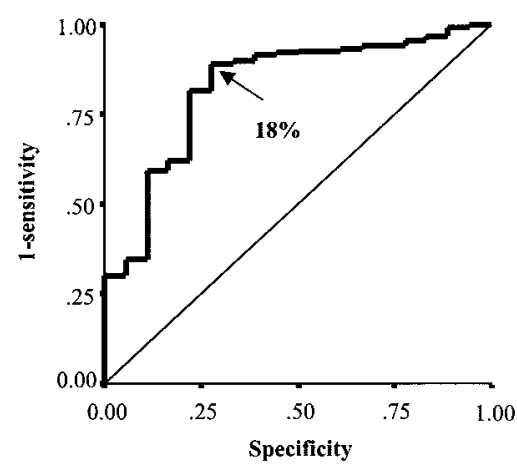

Figure 2. Receiver operating characteristics curve to predict the occurrence of postextubation stridor by measurement of cuff leak volume. A value of $18 \%$ was considered as a cutoff point, with sensitivity of $85.3 \%$ and specificity of $72.2 \%$.

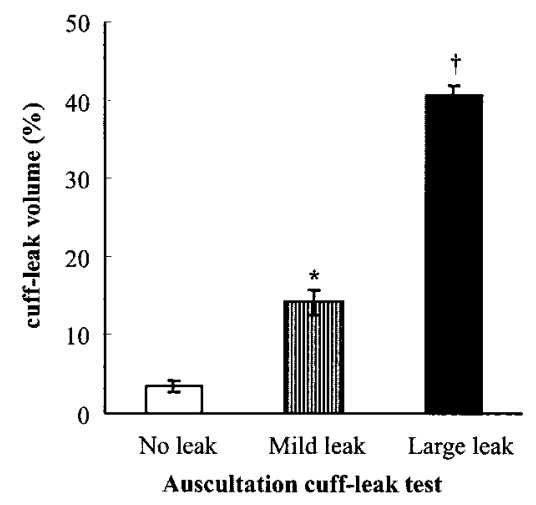

Figure 3. Relationship between auscultation cuffleak test and cuff leak volume (\%). No leak, no sound of leak was heard by using stethoscope detection; mild leak, leak was heard by using stethoscope; large leak, sound of leak was heard without using stethoscope. ${ }^{*} p=.007$, small leak vs. no leak; $p<.001$, large leak vs. small leak and no leak.

compared with attending physicians and anesthesiologists, but there was no difference with respect to the background training of operators before patient randomization into treated and nontreated groups (Table 1).

A number of other variables including the use of sedation, Glasgow Coma Scale (GCS), Acute Physiology and Chronic Health Evaluation II score, gender, and age were analyzed to identify the most sensitive risk factors contributing to the development of postextubation stridor. It is suggested that nonsedation treatment, low GCS score, and being female were risk factors to develop postextubation stridor in the untreated patients $(\mathrm{n}=236$, including patients in the nonintervention arm $[\mathrm{n}=$ 193] and the control group [ $n=43]$ in the intervention arm) (Table 2).

Table 1. Patients' characteristics and intratracheal intubation operators in the intervention arm

\begin{tabular}{|c|c|c|c|c|}
\hline & Control $(\mathrm{n}=43)$ & $1 \mathrm{INJ}(\mathrm{n}=42)$ & $4 \mathrm{INJ}(\mathrm{n}=42)$ & $p$ Value \\
\hline Gender, $\mathrm{M} / \mathrm{F}^{a}$ & $15 / 28$ & $15 / 28$ & $19 / 23$ & .523 \\
\hline Age, yrs ${ }^{b}$ & $68 \pm 16.1$ & $63.1 \pm 16$ & $67.3 \pm 17.6$ & .333 \\
\hline APACHE $\mathrm{II}^{b}$ & $18.3 \pm 14.2$ & $17.3 \pm 7$ & $17.0 \pm 5.3$ & .638 \\
\hline Glasgow Coma Scale ${ }^{b}$ & $10.2 \pm 3.9$ & $9.0 \pm 3.9$ & $9.6 \pm 3.5$ & .394 \\
\hline Duration of intubation, hrs ${ }^{b}$ & $169.5 \pm 98.8$ & $175.7 \pm 93.5$ & $149.8 \pm 91.8$ & .425 \\
\hline Endotracheal tube depth, $\mathrm{cm}^{b}$ & $21.3 \pm 1.7$ & $21.4 \pm 1.6$ & $21.4 \pm 1.5$ & .934 \\
\hline Patients source ${ }^{a}$ & & & & .183 \\
\hline Medical ICU & 25 & 17 & 18 & \\
\hline Surgical ICU & 18 & 27 & 24 & \\
\hline Intubation operator $^{a}$ & & & & .102 \\
\hline Attending physician & 10 & 3 & 5 & \\
\hline Resident & 22 & 28 & 25 & \\
\hline Anesthesiologist & 5 & 8 & 11 & \\
\hline Unknown prior to admission & 6 & 4 & 1 & \\
\hline
\end{tabular}

Control, patients received placebo; 1INJ, patients received one injection of sodium succinate; 4INJ, patients received four injections of the drug.

${ }^{a}$ Fisher's exact test; ${ }^{b}$ analysis of variance.

Table 2. Risk factors of developing postextubation stridor in untreated patients

\begin{tabular}{lll}
\hline & Crude OR $(95 \%$ CI $)$ & $p$ Value \\
\hline Sedation & & \\
Yes & 1.00 & .034 \\
No & $3.40(1.10,10.54)$ & \\
Gender & 1.00 & .0004 \\
Male & $6.42(2.30,17.96)$ & \\
Female & 1.00 & .114 \\
GCS & $4.04(0.71,22.87)$ & .017 \\
$13-15$ & $6.91(1.42,33.70)$ & \\
$9-12$ & 1.00 & .422 \\
$3-8$ & $0.41(0.05,3.61)$ & .287 \\
Age, yrs & $1.96(0.57,6.72)$ & .076 \\
$<60$ & $3.11(0.89,10.85)$ & .797 \\
$60-69$ & $1.00(1.00,1.00)$ & \\
$70-79$ & 1.00 & .762 \\
$\geq 80$ & $0.86(0.32,2.30)$ & \\
Intubation time, hrs & & \\
Endotracheal tube size, Fr & 1.00 & .101 \\
$\leq 7.0$ & $3.84(0.77,19.18)$ & .18 \\
$7.5-8.0$ & $3.15(0.59,16.86)$ & .019 \\
Intubation unit & $8.5(1.43,50.54)$ & .299 \\
Operating room & $0.00(0.00)$ & \\
Emergency room & $1.06(0.95,1.17)$ & \\
ICU & & \\
General ward & & \\
Prior to admission & & \\
APACHE II & &
\end{tabular}

OR, odds ratio; CI, confidence interval; GCS, Glasgow Coma Scale; ICU, intensive care unit; APACHE, Acute Physiology and Chronic Health Evaluation.

Data from the untreated patients of the nonintervention arm $(n=193)$ and the control group of the intervention arm $(n=43)$.

Intervention Arm. The patients were similar in age, gender, Acute Physiology and Chronic Health Evaluation II score, duration of intubation, endotracheal tube size, and types of ICU (medical vs. surgical) where the patients were treated (Table 1).

In the placebo group, approximately a $4 \%$ increase from baseline in CLV was observed at $24 \mathrm{hrs}$. In the groups treated with methylprednisolone, a significant increase from baseline in CLV was seen after the second and the fourth injection (both $p<.05$ ) in the 4INJ group and after the second injection in the 1INJ group $(p<.05$, Fig. 4).

We found that $18 \%$ of CLV was the optimal predictor for stridor development; we then analyzed the frequency of increased CLV $>18 \%$ from baseline after each injection of methylprednisolone, although the decision of extubation was not based on the values of measured CLV. 


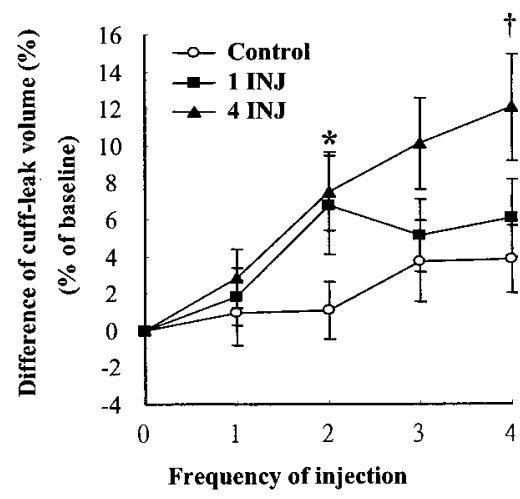

Figure 4. Differences in cuff leak volume (\%) changes with frequency of injections. Control group received four injections of normal saline every $6 \mathrm{hrs}$; the one injection of methylprednisolone (IINJ) group received an initial injection of methylprednisolone followed by three injections of normal saline; and the 4INJ group received four injections of methylprednisolone. $* p<.05$ vs. control group; $\dagger p<.05$ 4INJ group vs. control group.

Table 3. Frequency of increased cuff leak volume $>18 \%$ after each injection from baseline

\begin{tabular}{lcrcr}
\hline & \multicolumn{4}{c}{ Number of Injections } \\
\cline { 2 - 5 } & 1st & 2nd & 3rd & 4th \\
\hline Control & 11 & 8 & 13 & 14 \\
1INJ & 13 & 19 & 16 & 17 \\
4INJ & 16 & 40 & 53 & 60
\end{tabular}

Control, patients received placebo; 1INJ, patients received one injection of the drug; 4INJ, patients received four injections of the drug.

Data are reported as percent patients with increase in cuff leak volume $>18 \%$ from baseline (prior to injection of methylprednisolone sodium succinate).

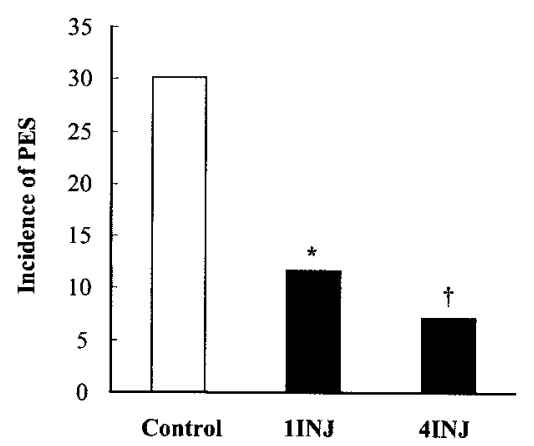

Figure 5. Incidence of postextubation stridor $(P E S)$ in the intervention arm (cuff leak volume $<24 \%)$ of the study. ${ }^{*} p=.015$, 1INJ group vs. control group; $\dagger p=.005$, 4INJ group vs. control group. IINJ, one injection of methylprednisolone; $4 I N J$, four injections.

Table 4. Intubation duration and intensive care unit (ICU) stay in relation to Glasgow Coma Scale (GCS) score in the intervention arm

\begin{tabular}{lccc}
\hline \multicolumn{1}{c}{ GCS } & $3-8(\mathrm{n}=51)$ & $9-15(\mathrm{n}=55)$ & $p$ Value \\
\hline Intubation duration, hrs & $178.5 \pm 85.0$ & $149.2 \pm 88.81$ & .086 \\
Stridor & 10 & 4 & .061 \\
$\quad$ Control & 6 & 3 & \\
IINJ & 2 & 0 & \\
$\quad$ 4INJ & 2 & 1 & .485 \\
ICU stay, days & $12.4 \pm 6.5$ & $11.4 \pm 7.7$ & .701 \\
Hospital stay, days & $30.3 \pm 12.8$ & $31.6 \pm 21.4$ & \\
\hline
\end{tabular}

Control, patients received placebo; 1INJ, patients received one injection of sodium succinate; 4INJ, patients received four injections of the drug.

As many as $19 \%$ patients had increased CLV $>18 \% 12$ hrs after treatment in the 1INJ group, and $40-60 \%$ of patients showed increased CLV $>18 \%$ during a prolonged treatment in the $4 \mathrm{INJ}$ group (Table 3).

The changes in CLV were consistent with the incidence of postextubation stridor. As many as $30.2 \%$ of patients developed postextubation stridor in the placebo group compared with an incidence as low as $7.1 \%$ and $11.6 \%$ in the $4 \mathrm{INJ}$ group and the 1INJ group, respectively (both $p<.05$, Fig. 5). The rate of reintubation was $18.6 \%$ (eight of 43 ) in the placebo group compared with $7.1 \%$ (three of 42) in the 4INJ group and 4.7\% (two of 43) in the 1INJ group (both $p<$ $.05)$. Neither the CLV nor the incidence of postextubation stridor was different between the two treated groups (Fig. 1).

Since a low GCS was associated with an increased risk of stridor, one may be concerned that exposure of this population of patients to the present protocol could place them at a risk of inhalation. Importantly, our data showed no difference in the lengths of ICU stay and hospital stay between the small size groups of patients with low and high GCS on completion of the study protocol (Table 4).

\section{DISCUSSION}

Endotracheal intubation is a useful approach to manage critically ill patients who may or may not require mechanical ventilation. However, this intervention may induce laryngeal mucosa trauma as a result of mucosa abrasion and necrosis secondary to compression, which leads to postextubation stridor $(2,3,16,17)$. Histologic findings of postextubation stridor are characterized with mucosal inflammation, mucosal ulceration, and edema (18). Corticosteroids have been used in infants and children to prevent or reduce postextubation stridor by suppressing mucosal inflammation including inhibition of leukocyte migration, maintenance of cell membrane integrity, attenuation of lysosome release, and reduction of fibroblast proliferation $(19,20)$ and tissue swelling (21). However, intravenous infusion of $\beta$-methasone had no protective effect in attenuation of postextubation inflammation in pediatric ICU (22). In experimental settings, dexamethasone effectively suppressed postintubation laryngeal edema in monkeys (23).

There are few data in adult patients compared with pediatric patients with respect to the use of corticosteroids. A bolus intravenous injection of dexamethasone or hydrocortisone $1 \mathrm{hr}$ before extubation failed to prevent laryngeal edema in adults $(4,12)$. The discrepancies observed in these previous studies could be due to several factors including age, inclusion criteria, duration of intubation, dose, timing and length of treatment, and risk levels of developing stridor.

Cuff leak volume has been considered as a good indicator to predict the occurrence of postextubation stridor. Sandhu et al. (9) found that of 110 patients studied, all 13 patients (12\%) who developed postextubation stridor had a CLV ranging from $0 \%$ to $24 \%$. Their data are supported by the present study, where the incidence of postextubation stridor was $2.6 \%$ (five of 193 patients) if CLV was $\geq 24 \%$ and increased to $30.2 \%$ (13 of 43 patients) with CLV $<24 \%$. The difference ( $12 \%$ vs. $30 \%)$ in the incidence of postextubation stridor seen at an identical cuff leak cutoff point of $<24 \%$ between the Sandhu et al. and the present study may be explained by a population difference, because only trauma patients were included in the former study and all kinds of ICU patients were included in the present study. Thus, the different inci- 
dences of postextubation stridor seen in the studies may reflect the severity of illness. Taken together, these observations suggest that examination of CLV is a useful approach to identify patients at high risk of developing postextubation stridor.

In the present study, a CLV of $18 \%$ was the best cutoff value to predict postextubation stridor based on its $85.3 \%$ of sensitivity at $18 \%$ CLV compared with $79.7 \%$ of sensitivity at $24 \%$ CLV. However, there is no significant difference in the specificity $(72.2 \%$ vs. $77.8 \%)$ between the CLVs at $18 \%$ and $24 \%$. Furthermore, although the CLV $>24 \%$ is very useful in predicting the absence of stridor and CLV $<24 \%$ identifies a high-risk group, $70 \%$ of control participants did not develop stridor. We speculate that this gap seen between the predicted high incidence and the actual low rate might be due to a number of potential mechanisms including secretions adherent to the outside of the endotracheal tube and differences in measurements between inspired tidal volume and inhaled tidal volume around the endotracheal tube.

Other studies reported CLVs of $10 \%$ and $12 \%$ as cutoff points to predict the development of postextubation stridor $(5$, $8-10)$. The difference in cutoff points among the studies may be due to differences in tidal volume used during measurement of cuff leak, as we used a tidal volume of $8 \mathrm{~mL} / \mathrm{kg}$ compared with a tidal volume of $10-12 \mathrm{~mL} / \mathrm{kg}$ in other studies (5, 8-10). We demonstrated an excellent correlation between the measurements of auscultation cuff leak test and CLV. The former technique appears to be more convenient to perform at bedside and is without risk of developing aspiration pneumonia.

Treatment with intravenous injection of methylprednisolone sodium succinate resulted in a significant increase in CLV after the second injection both in the 4INJ group and in the 1INJ group where normal saline was used as a second injection. This observation suggests that a) a time frame of 6-7 hrs after a single injection is required to exert the protective effect of methylprednisolone in reducing the incidence of postextubation stridor; and b) injections with either four separate doses or a single initial dose of methylprednisolone do not make any significant difference in the incidence of postextubation stridor. We do not anticipate any significant changes in respiratory mechanics over the period of cuff leak tests after each injection of methylprednisolone, since the conditions of the patients were relatively stable, where all patients met the extubation criteria before being included in the current protocol. We credit the protective effect of methylprednisolone demonstrated in the present study to two major factors: the homogeneous selection of patient population by using the cuff-leak test, and a prolonged therapy with methylprednisolone compared with previous studies by other investigators $(4,12)$.

A few risk factors were identified in the present study including the use of sedation before intubation, GCS score, and gender. First, the use of sedation in advance of intubation may help reduce patients' anxiety that would have caused them to fight against intubation, resulting in vocal cord damage (24), as the quality of endotracheal intubation can contribute to laryngeal hoarseness and vocal cord squeal (25). Second, a low GCS score (3-8 points) was associated with a high incidence of postextubation stridor, which may result from a reduced ability to cough and a more frequent requirement of intratracheal suction. Third, consistent with our results, several other investigators also reported that female patients had a higher risk to develop stridor compared with male patients $(4,21$, 26). This may be related to anatomy, as the larynx is smaller, the mucosal coverage is thinner, and the mucosal membrane is less resistant to trauma in female than in male subjects $(12,27)$.

The side effects with steroid therapy over 24 hrs are minimal (21). In the present study there were no obvious complications such as gastrointestinal bleeding, high blood sugar, or increased risk of infection.

\section{CONCLUSIONS}

Our study reveals that the use of a cutoff point of CLV can help identify patients who have a high risk of developing postextubation stridor. Either a single or multiple injections of methylprednisolone 6 hrs before extubation can effectively reduce the incidence of postextubation stridor in critically ill adult patients.

\section{ACKNOWLEDGMENT}

We thank Chin-Li Lu for statistical assistance.

\section{REFERENCES}

1. Natanson C, Shelhamer JH, Parrillo JE: Intubation of the trachea in the critical care setting. JAMA 1985; 253:1160-1165

2. Stauffer JL, Olson DE, Petty TL: Complications and consequences of endotracheal intubation and tracheotomy. A prospective study of 150 critically ill adult patients. Am J Med 1981; 70:65-76

3. Kastanos N, Estopa Miro R, Marin Perez A, et al: Laryngotracheal injury due to endotracheal intubation: Incidence, evolution, and predisposing factors. A prospective longterm study. Crit Care Med 1983; 11:362-367

4. Ho LI, Harn HJ, Lien TC, et al: Postextubation laryngeal edema in adults. Risk factor evaluation and prevention by hydrocortisone. Intensive Care Med 1996; 22:933-936

5. Jaber S, Chanques G, Matecki S, et al: Postextubation stridor in intensive care unit patients. Risk factors evaluation and importance of the cuff-leak test. Intensive Care Med 2003; 29:69-74

6. Epstein SK, Ciubotaru RL: Independent effects of etiology of failure and time to reintubation on outcome for patients failing extubation. Am J Respir Crit Care Med 1998; 158:489-493

7. Demling RH, Read T, Lind LJ, et al: Incidence and morbidity of extubation failure in surgical intensive care patients. Crit Care Med 1988; 16:573-577

8. Marik P: The cuff-leak test as a predictor of postextubation stridor: A prospective study. Respir Care 1996; 41:509-511

9. Sandhu RS, Pasquale MD, Miller K, et al: Measurement of endotracheal tube cuff leak to predict postextubation stridor and need for reintubation. J Am Coll Surg 2000; 190: 682-687

10. Miller RL, Cole RP: Association between reduced cuff leak volume and postextubation stridor. Chest 1996; 110:1035-1040

11. Fisher MM, Raper RF: The "cuff-leak" test for extubation. Anaesthesia 1992; 47:10-12

12. Darmon JY, Rauss A, Dreyfuss D, et al: Evaluation of risk factors for laryngeal edema after tracheal extubation in adults and its prevention by dexamethasone. A placebocontrolled, double-blind, multicenter study. Anesthesiology 1992; 77:245-251

13. Anene O, Meert KL, Uy H, et al: Dexamethasone for the prevention of postextubation airway obstruction: A prospective, randomized, double-blind, placebo-controlled trial. Crit Care Med 1996; 24:1666-1669

14. Daley BJ, Garcia-Perez F, Ross SE: Reintubation as an outcome predictor in trauma patients. Chest 1996; 110:1577-1580

15. Chevron V, Menard JF, Richard JC, et al: Unplanned extubation: Risk factors of development and predictive criteria for reintubation. Crit Care Med 1998; 26:1049-1053

16. Bishop MJ, Weymuller EA Jr, Fink BR: Laryngeal effects of prolonged intubation. Anesth Analg 1984; 63:335-342

17. Bishop MJ: Mechanisms of laryngotracheal 
injury following prolonged tracheal intubation. Chest 1989; 96:185-186

18. Way WL, Sooy FA: Histologic changes produced by endotracheal intubation. Ann Otol 1965; 74:799-813

19. Demling W, Oech SR: Steroid and antihistaminic therapy for postintubation subglottic edema. Anesthesiology 1961; 22:933-936

20. Haynes RC: Adrenocorticotropic hormones, adrenocortical steroids and their synthetic analogues; inhibitors of the synthesis and actions of adrenocortical hormones. In: The Pharmacological Basis of Therapeutics. Gilman
AG, Rall TW, Nies AS (Eds). New York, Pergamon Press, 1990, pp 1431-1462

21. Hawkins DB, Crockett DM, Shum TK: Corticosteroids in airway management. Otolaryngol Head Neck Surg 1983; 91:593-596

22. Goddard JE Jr, Phillips OC, Marcy JH: Betamethasone for prophylaxis of postintubation inflammation. A double-blind study. Anesth Analg 1967; 46:348-353

23. Biller HF, Bone RC, Harvey JE, et al: Laryngeal edema: An experimental study. Ann Otol Rhinol 1970; 174:1084-1087

24. Sivilotti ML, Filbin MR, Murray HE, et al:
Does the sedative agent facilitate emergency rapid sequence intubation? Acad Emerg Med 2003; 10:612-620

25. Mencke T, Echternach M, Kleinschmidt S, et al: Laryngeal morbidity and quality of tracheal intubation: A randomized controlled trial. Anesthesiology 2003; 98:1049-1056

26. Gaynor EB, Greenberg SB: Untoward sequelae of prolonged intubation. Laryngoscope 1985; 95:1461-1467

27. Whited RE: A prospective study of laryngotracheal sequelae in long-term intubation. Laryngoscope 1984; 94:367-377 